\title{
Role of exosomes in pancreatic cancer (Review)
}

\author{
YUNMENG YAN, GUANGZHEN FU and LIANG MING \\ Department of Clinical Laboratory and Key Clinical Laboratory of The Henan Province, \\ The First Affiliated Hospital of Zhengzhou University, Zhengzhou, Henan 450052, P.R. China
}

Received May 4, 2017; Accepted November 20, 2017

DOI: $10.3892 / 01.2018 .8348$

\begin{abstract}
Pancreatic cancer is one of the most lethal malignancies. Exosomes, which are released by multiple cell types, such as cancer cells, contain functional biomolecules (including proteins, nucleic acids and lipids) that can be horizontally delivered to recipient cells. Exosomes act as the most prominent mediator of intercellular communication and can regulate, instruct and re-educate their surrounding microenvironment and target specific organs. The present review performed an extensive search of multiple databases from 2005 to April 23 2017, for eligible literature relating to exosomes and their role in pancreatic cancer. With a focus on the latest findings for pancreatic cancer exosomes, their role in tumorigenesis was summarized, as well as their aggressive behaviors and their contribution to immunosuppression and therapy resistance in pancreatic cancer. In addition, the potential function of exosomes as novel diagnostic biomarkers is briefly discussed. Finally, we propose potential clinical applications for exosomes in pancreatic cancer.
\end{abstract}

\section{Contents}

1. Introduction

2. Components and functions of exosomes

3. The role of exosomes in the pathogenesis of pancreatic cancer

4. Exosomes regulate therapy resistance in pancreatic cancer

Correspondence to: Professor Liang Ming, Department of Clinical Laboratory and Key Clinical Laboratory of The Henan Province, The First Affiliated Hospital of Zhengzhou University, 1 Jianshe Road, Zhengzhou, Henan 450052, P.R. China

E-mail: mingliang3072@163.com

Abbreviations: MIF, migration inhibitory factor; TLR4, toll-like receptor 4; DCs, dendritic cells; CIKs, cytokine-induced killer cells; CAFs, cancer-associated fibroblast; IAP, inhibitors of apoptosis; CP, chronic pancreatitis; GPC1, glypican-1; IPMN, intraductal papillary mucinous neoplasm; CA19-9, carbohydrate antigen 19-9

Key words: exosomes, pancreatic cancer, distant metastasis, tumor biomarkers, prognosis, cancer therapy
5. Exosomal miRNAs and proteins for pancreatic cancer diagnosis

6. Conclusion

7. Future perspectives

\section{Introduction}

Pancreatic cancer is recognized as a highly malignant and incurable disease worldwide. In 2016, an estimated 53,070 new cases were diagnosed and 41,780 people eventually died from pancreatic cancer, making it the fourth leading cause of cancer deaths in the USA. Although the survival rate has increased for most cancers, improvements have been marginal for patients with pancreatic cancer; the 5-year relative survival is merely $8 \%$ (1). The dismal prognosis for pancreatic cancer is attributed to multiple factors, such as the advanced stage of initial detection, the high risk for distant metastasis, and a subpar response to conventional therapies. Sequencing the genomes of pancreatic cancers indicated 12 important signaling pathways that do not promote distant metastasis until nearly 20 years after the initial mutation occurred. This evidence has offered a time frame for pancreatic carcinogenesis, suggesting that a large window of opportunity may exist for early detection, which could improve the prognosis of this lethal disease $(2,3)$. Thus, further studies are required into the molecular pathology that orchestrates the process of pancreatic tumorigenesis, in order to improve personalized diagnostics and therapeutic regimens.

Exosomes, which are secreted by multiple cell types and by cancer cells, contain functional biomolecules (including proteins, nucleic acids and lipids) that can be horizontally delivered to recipient cells. They are distinguished from apoptotic bodies and microvesicles by their heterogeneous size, origin and composition (4,5). Exosomes (30-150 nm in diameter) are extracellular vesicles generated by the inward budding of the membrane (endocytosis), which leads to the subsequent formation of multivesicular bodies and their release by exocytosis. Thus, on their membranes, exosomes express a variety of markers of multivesicular bodies, such as tetraspanins. Ectosomes (0.1-2 $\mu \mathrm{m}$ in diameter) are formed by outward blebbing from the plasma membrane and are subsequently released by proteolytic cleavage from the cell surface, thus expressing antigens of the origin cell. Apoptotic bodies (1-4 $\mu \mathrm{m}$ in diameter) are generated upon apoptotic cell death $(6,7)$. 
Exosomes are the most prominent mediators of intercellular communications that regulate, instruct, and re-educate their surrounding microenvironment and target organs $(8,9)$. The interaction between exosomes and recipient cells is mediated by the membrane proteins of exosomes, such as the phosphatidylserine and proteoglycan receptors $(9,10)$. Additionally, the presence of particular tetraspanins or integrins on the exosomal membrane is likely to play a vital role in selectively acting on their target cells or organs $(11,12)$. Exosome-associated diagnosis and follow-up has been extensively promoted due to the easy isolation and identification of exosomes in body fluids, especially the peripheral blood (13). Furthermore, Li et al (14) identified >1,000 circular RNAs (circRNAs) in human plasmatic exosomes. Their study indicated the potential of circRNAs as a novel exosome-associated tumor biomarker and discussed the molecular functions of exosomal circRNAs.

\section{Components and functions of exosomes}

A systematic search of ExoCarta (http://www.exocarta.org/), EVpedia (http://student4.postech.ac.kr/evpedia2_xe/xe/) and Vesiclepedia (http://microvesicles.org/index.html\#) was performed to select the most important components of exosomes. The top five most common identified exosomal proteins were $\mathrm{CD} 9$, heat shock protein family A member 8 (HSPA8), programmed cell death 6 interacting protein (PDCD6IP), glyceraldehyde-3-phosphate dehydrogenase (GADPH), and actin $\beta$ (ACTB); the majority of these are known to be implicated in the biogenesis, sorting and release of exosomes. The simple structure and components of exosomes are summarized in Fig. 1.

Lipids. Exosomes consist of a lipid bilayer, and transmembrane proteins have crucial roles in the development of a mosaic model. Lipids exert a vital function in maintaining the rigidity and stability of the exosomal membrane, as well as in the processes of fusion and budding. Exosomal RNA is well-protected in exosomes due to the integrity of the membrane (15). In particular, exosomal membranes have abundant phosphatidylserine, monosialodihexosylganglioside cholesterol and sphingomyelin (16). Exosomal lipids can carry other lipids with biological activities, as well as second messengers, such as phosphatidic acid, ceramides and diglycerides, that are implicated in exosome biogenesis. Beloribi et al (17) demonstrated that exosomal lipids initiated apoptosis in human pancreatic carcinoma SOJ-6 cells by inhibiting the Notch-1 pathway. Exosomal lipids also led to drug resistance in human pancreatic cancer MiaPaCa-2 cells via the $\mathrm{C}-\mathrm{X}-\mathrm{C}$ motif chemokine receptor 4 (CXCR4)/stromal cell derived factor (SDF)-1 $\alpha$ signaling axis, which implied that exosomal lipids might function in the progression and drug resistance of pancreatic cancer (18). At present, there is only one study on prostate cancer that indicates that exosomal lipids have the potential to serve as cancer biomarkers (19). It is possible that progress in lipidomics will aid in the improved understanding of the biological mechanisms of exosomal formation, sorting and release under physiological and pathological conditions.

Proteins. Several studies on exosomes from multiple organs/cell types and cell lines, under physiological and pathophysiological conditions, have demonstrated the enrichment of proteins in exosomes (20-22). Exosomal proteins differ vastly by the type and state of the cell the exosomes were derived from, suggesting that they may be ideal candidates for cancer biomarkers. To date, $>7,000$ proteins have been identified through significant technical improvements in mass spectrometry. Proteomic analysis of exosomes from multiple tissues indicate that exosomes are comprised of some common vesicular proteins, such as tetraspanins [CD9, CD82, CD81, CD63, CD151 and tetraspanin 8 (Tspan8)], which are constituent components of exosomes $(23,24)$. The tetraspanins CD151 and Tspan8 are exosome components that are critical for the interactions between tumor-initiating cells and the tumor microenvironment (25). Cytoplasmic and plasma membrane proteins are more commonly released into exosomes than mitochondrial and nuclear proteins, which indicates that membrane proteins have great potential to be candidates for protein markers $(26,27)$.

Exosomes also contain cell type-specific proteins, which are particularly useful for the identification of specific surface biomarkers. Most of the cell type-specific surface biomarkers have been validated as cancer and cancer stem cell-associated markers. Among them, melanoma tumor exosomes express the tumor-related protein melanoma antigen recognized by $\mathrm{T}$ cells 1 (MART1), while epithelial cell-originated tumor exosomes express the epithelial cell adhesion molecule (EpCAM) $(27,28)$. Glioblastoma tumor exosomes express epidermal growth factor receptor (EGFR) variant III (29) and the tumor exosomes of docetaxel-resistant prostate cancer cells express the multidrug resistance gene 1 (MDR-1) (30).

Furthermore, it has been proposed that mutations in $>1 \%$ of the human genome are correlated with carcinogenesis; mutant proteins, such as mutant KRAS, the oncoprotein MET and tissue factor proteins that uniquely appear in pathological cells, have been validated to be released via exosomes (31-33). EGFR is secreted by five pancreatic cancer cell lines in exosomes as two distinct EGFR forms. The identification of EGFR as a plasma biomarker and a personalized therapeutic is promising in pancreatic cancer (34).

Nucleic acids. Exosomes also contain microRNA (miRNAs), tRNA, rRNA mRNA, DNA, and the recently identified circRNA (14,35-37). Exosomes interact with their target cells mainly via membrane fusion and transfer of exosomal contents. miRNAs could deliver biological information that regulates the function of target cells.

miRNAs are 19-25 nt long non-coding RNAs that repress the stability and translation of mRNAs, controlling genes implicated in many cellular processes. They have important roles in the mediation of nearly all signaling pathways within a cell, and their dysfunction is associated with the initiation, progression and metastasis of cancer, including pancreatic cancer (38-40). Furthermore, miRNA-155 was validated to control exosome synthesis and to promote gemcitabine resistance in pancreatic cancer. Targeting therapy to miR-155 or exosome secretion effectively attenuated tumor chemoresistance, representing a novel therapeutic target for gemcitabine treatment in pancreatic cancer (41). In pancreatic cancer, Zinc finger E-box-binding homeobox (ZEB)1 and ZEB2 are important mediators of the 


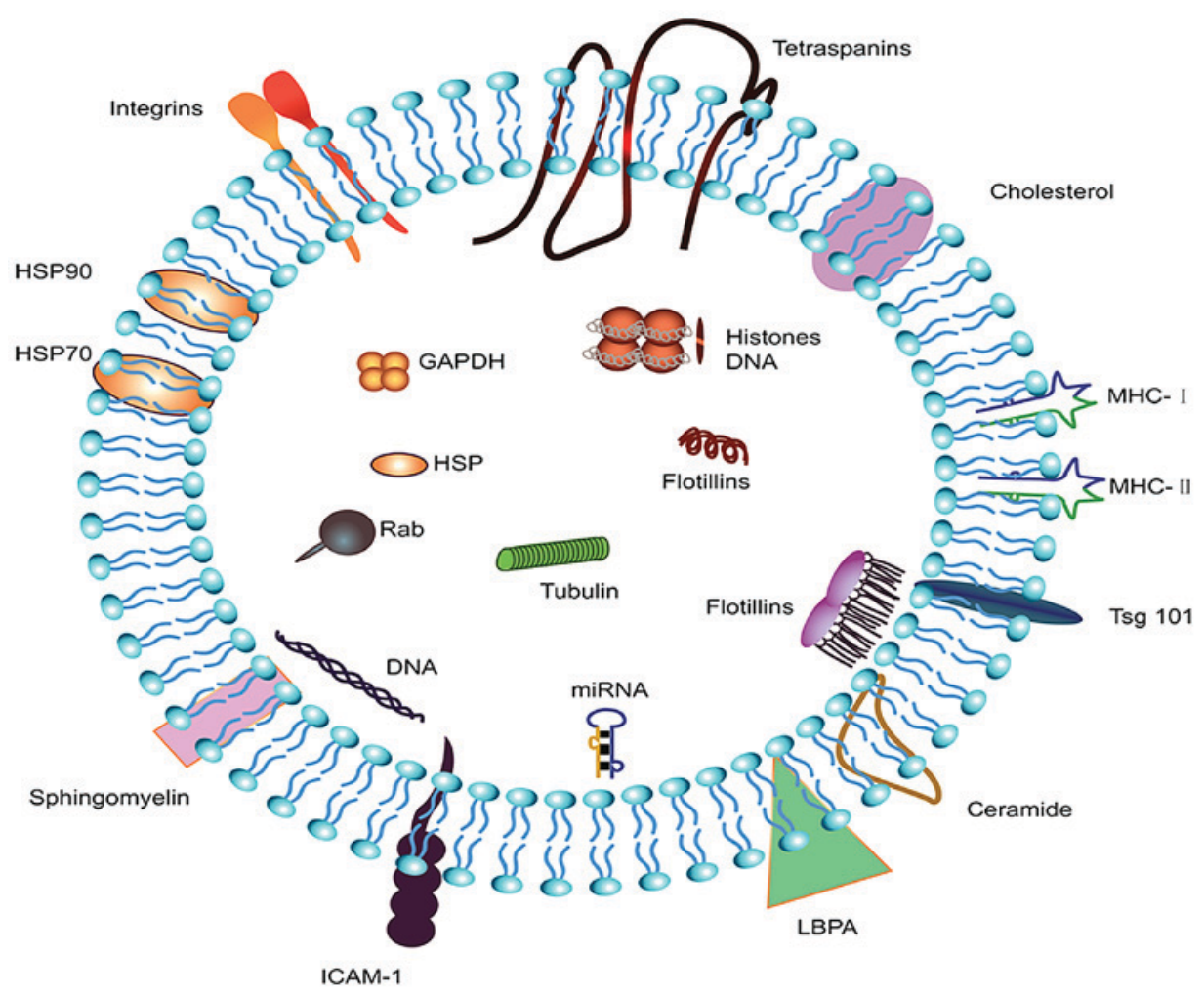

Figure 1. Components and structure of exosomes. Exosomes are small vesicular structures carrying various pathogenic miRNAs, mRNAs, DNA fragments, and proteins. The top 5 most commonly identified exosomal proteins are CD9, HSPA8, PDCD6IP, GADPH, and ACTB. HSPA8, heat shock protein family A member 8; PDCD6IP, programmed cell death 6 interacting protein; GAPDH, glyceraldehyde-3-phosphate dehydrogenase; ACTB, actin $\beta$; HSP, heat shock protein; ICAM-1, intercellular adhesion molecule 1; MHC, major histocompatibility complex; LBPA, lysobisphosphatidic acid.

epithelial-to-mesenchymal transition and maintain stemness by suppressing the miR-200 family, thereby promoting migration (42). Manier et al (43) identified miRNAs as the most prominent small RNAs present in exosomes isolated from the serum of multiple myeloma patients and healthy controls by using small RNA sequencing on circulating exosomes. Two of these miRNAs, namely, let-7b and miR-18a, were significantly associated with both progression-free survival and overall survival in a univariate analysis. These findings support the use of circulating exosomal miRNAs to improve the identification of patients with newly diagnosed multiple myeloma with poor prognosis. The presence of miRNAs in numerous body fluids, especially serum, correlates with the recovery of solid cancers and hematologic malignancies and may be valuable for the early diagnosis of multiple types of cancer, potentially offering superior performance over conventional methods for cancer diagnosis $(44,45)$.

Representing a novel type of endogenous non-coding RNAs, circRNAs have gained attention recently for their involvement in multiple biological processes. CircRNAs are ubiquitously expressed in eukaryotic cells and modulate gene expression by acting as sponges of miRNAs or proteins, such as RNA-binding proteins (RBPs) $(46,47)$. Li et al (14) demonstrated that circRNA is enriched and stable in exosomes, identified up to 1,000 serum exosomal circRNAs, and discriminated between patients with colon cancer and healthy controls; expression patterns of circ-KLDHC10 were dramatically enhanced in serum from cancer patients. Their finding that serum exosomal circRNAs could discriminate patients with cancer from normal controls suggests exosomal circRNAs have great translational potential as a circulating biomarker for the early detection of cancer.

Recently, IncRNAs were demonstrated to exist in exosomes, and they could function as potential stable biomarkers for cancer patients. Li et al (48) have found that plasma Long intergenic non-protein-coding RNA 152 (LINC00152) in exosomes was a potential biomarker and highly stable in patients with gastric cancer. A similar result revealed that the exosomal transfer of the lncRNA mediator of reprogramming (linc-RoR) may be involved in hepatocellular carcinoma progression and treatment failure (49). These findings demonstrate that the components of serum-derived exosomes can be utilized to predict, treat and evaluate therapy resistance for many cancers.

\section{The role of exosomes in the pathogenesis of pancreatic cancer}

Exosomes engage in tumorigenesis and the aggressive behavior of pancreatic cancer. Invasion is one of the primary features of pancreatic cancer, and failures in pancreatic cancer therapy are generally due to metastasis, because distant lesions are generally resistant to most chemotherapeutic agents. Metastasis is a complicated, sequential event and is a sign of malignant cancer. Metastasis-initiating cells that disseminate from invasive carcinomas disperse via the blood or lymphatic vessels. Circulating tumor cells that survive in the bloodstream subsequently migrate to target tissues. Infiltrated cells in the new microenvironment can survive chemoradiotherapy 
and initiate cancer regrowth, leading to cancer recurrence and metastasis (50). These steps are supported by the complex interaction of cancer cells with the tumor stroma that involves multiple intercellular communications pathways $(51,52)$.

Recently, there has been an increase in the number of studies demonstrating the important function exerted by exosomes in cancer initiation, progression and distant metastasis. For instance, exosomes have been verified to have the ability to transform normal cells into cancer cells in breast and prostate cancer $(53,54)$. Exosomes promote cancer cell proliferation by delivering carcinogenic proteins, cell-cycle-associated mRNAs, specific miRNAs, cytokines and adhesion molecules to cancer cells (55-57). They can deliver tumor suppressor miRNA, such as miR-200, to confer metastatic properties and promote breast cancer cell metastasis (58). Cancer cell-derived exosomes can also activate the phosphoinositide 3-kinase (PI3K)/AKT serine/threonine kinase 1 (Akt) and mitogen-activated protein kinase (MAPK) pathways to facilitate the proliferation of gastric cancer cell lines (59). However, a different result was obtained in pancreatic cancer cells, where exosome lipids led to cancer cell apoptosis via the repression of the Notch-1 survival signaling pathway (17). Another study (18) in pancreatic cancer cell line, MiaPaCa-2, revealed that exosomal lipids could induce the activation of nuclear factor (NF)-kB, which facilitated the expression and release of SDF- $1 \alpha$. The binding of SDF-1 $\alpha$ and its receptor CXCR4 activated the Akt signaling pathway, inhibiting cell death (18). This finding suggested that exosomal lipids may be involved in pancreatic cancer development and drug resistance. Exosomes derived from pancreatic stellate cells contained abundant miR-21-5p and miR-451a. Exosome-mediated delivery of these components enhanced proliferation, migration and the specific gene expression of pancreatic cancer cells (60).

Costa-Silva et al (8) demonstrated that pancreatic cancer-derived exosomes could lead to liver pre-metastatic niche initiation in mice and could gradually increase the cancer niche burden. Primary pancreatic cancer-derived exosomes alter the pre-metastatic liver microenvironment by initiating an inflammatory response, which results in the preferential implantation of pancreatic cancer cells in the liver. Kupffer cells selectively absorb the exosomes released by pancreatic cancer and can increase the secretion of transforming growth factor $\beta$ (TGF $\beta$ ). TGF $\beta$ activates hepatic stellate cells and then upregulates fibronectin production. The fibrotic microenvironment induces the accumulation of bone marrow-derived cells, such as neutrophils and macrophages. These cells bind primarily to fibronectin-associated hepatic sites, consequently facilitating the initiation of the liver pre-metastatic niche. Further study revealed that the macrophage migration inhibitory factor (MIF) was highly abundant in pancreatic cancer-derived exosomes, which orchestrated the liver metastasis (8). Compared with subjects who had a bulky primary tumor, exosomal MIF was significantly upregulated in early stage pancreatic cancer patients who later developed distant liver metastasis. The identification of MIF pancreatic cancer-derived exosomes has opened novel avenues for the biological mechanisms of metastasis and might be a potential prognostic marker for the progression of pancreatic cancer metastasis.
An et al (61) and colleagues used proteomic analysis to quantify the protein level of serum exosomes from patients with locally advanced pancreatic cancer undergoing chemoradiotherapy. They isolated exosomes from the serum of 10 patients with locally advanced pancreatic cancer at serial time points over the course of therapy, and quantitative analysis was performed using the iTRAQ method. They detected approximately 700-800 exosomal proteins per sample, several of which have been implicated in metastasis and treatment resistance. They compared the exosomal proteome of patients at different time points during treatment to healthy controls and identified eight proteins that exhibited global treatment-specific changes. They then tested the effect of patient-derived exosomes on the migration of tumor cells and demonstrated that patient-derived exosomes, but not exosomes from healthy controls, induced cell migration, supporting their role in metastasis. Their data demonstrated that exosomes can be reliably extracted from patient serum and analyzed for protein content. The differential loading of exosomes during the course of therapy suggests that exosomes may provide novel insights into the development of treatment resistance and metastasis.

Exosome-mediated immunosuppression in pancreatic cancer. Emerging evidence has demonstrated that communications between tumor and immune cells through exosomes have a dynamic and complex role in regulating tumor immunity, which is determined by specific proteins and genetic components (62). During pancreatic cancer progression and distant metastasis, most cancer cells are suppressed by the immune response. Most patients with pancreatic cancer are in a condition of immune disorder or immunosuppression. Increasing studies point to the well-documented role of pancreatic cancer-derived exosomes in immunosuppression.

Representative literature suggests that pancreatic cancer-derived exosomes modulate the expression pattern of toll-like receptor 4 (TLR4) in dendritic cells (DCs) through miRNA-203. When absorbed by dendritic cells, these exosomes decreased TLR4 expression and the downstream associated cytokines, such as interleukin (IL)-12 and tumor necrosis factor (TNF)- $\alpha$. Thus, exosomes attenuate DC-mediated tumor suppressive responses initiated by TLR4 (63). Similarly, Ding et al (64) have demonstrated that pancreatic cancer-associated miRNAs could be delivered to DCs through exosomes and suppress target mRNA expression. Furthermore, pancreatic cancer-generated exosomes restrain regulatory factor $\mathrm{X}$-associated protein expression by means of miR-212-3p, which could decrease the expression level of major histocompatibility complex (MHC) II and result in the immune tolerance of DCs. However, pancreatic cancer-derived exosomal miRNAs can inhibit the tumor suppressive function of DC/cytokine-induced killer cells (CIKs), and miRNA-depleted exosomes may increase the tumor suppressive activity of DC/CIKs. In summary, pancreatic cancer-derived exosomes regulate host immunosuppression via regulating the function, maturation and differentiation of DCs.

Macrophages exhibit dramatic plasticity and alter their physiological state based on environmental conditions, especially the cancer microenvironment (65). A previous study has revealed that the content of pancreatic cancer-derived exosomes was altered by transfection with miR-155 
and miR-125b2, inducing differential communication and remodeling the macrophage polarization to the M1 phenotype (66). This alteration can cause an inhibitory effect on pancreatic cancer invasion and metastasis. Another study provides evidence that salivary exosomes from mice with pancreatic ductal adenocarcinoma exhibit a suppressive effect that results in reduced tumor-killing capacity by NK cells (67). Salivary exosomes from mice with pancreatic ductal adenocarcinoma where pancreatic tumors were engineered to suppress exosome biogenesis failed to suppress NK cell cytotoxicity against tumor cells, as opposed to salivary exosomes from mice with pancreatic ductal adenocarcinoma with normal tumor exosome biogenesis (67). According to these findings, immunotherapy aimed towards alterative manipulation of cancer cell-derived exosome components, such as miRNAs, may have therapeutic potential in treating pancreatic cancer.

Clinicopathological characteristics of pancreatic cancer associated with exosomes. Weight loss and new-onset diabetes are characteristics of the paraneoplastic effect of pancreatic cancer, and these phenomena precede the diagnosis of the disease (68). A previous study suggested that exosomal adrenomedullin serves a crucial role in the initiation of diabetes and lipolysis in adipocytes (69). The biological mechanism of pancreatic cancer-associated diabetes is complicated. A potential mechanism has been revealed where pancreatic cancer-derived exosomal adrenomedullin, triggers endoplasmic reticulum stress and dysfunction of the unfolded protein response, resulting in $\beta$-cell disorder and apoptosis. Further experiments demonstrated that exosome-mediated transfer of adrenomedullin into $\beta$-cells was regulated through micropinocytosis and caveolin-dependent endocytosis (70).

Adipose tissue lipolysis is partly responsible for weight loss, which is one of the alarming symptoms associated with pancreatic cancer. Exosomal adrenomedullin, a candidate mediator, promotes lipolysis in adipocytes by selectively targeting its receptor. This interaction induces the activation of the p38 and extracellular signal-regulated kinase (ERK)1/2 MAP kinase signaling pathways that can promote the phosphorylation of hormone-sensitive lipase, therefore facilitating lipolysis $(69,71)$.

\section{Exosomes regulate therapy resistance in pancreatic cancer}

Up to $74 \%$ of patients with pancreatic cancer who receive adjuvant therapy with gemcitabine ultimately present with rapid tumor regression and treatment failure (72). The elucidation of the detailed mechanisms that lead to chemotherapy resistance is of great importance for improving pancreatic cancer survival. Exosomes induce the progression of chemotherapy resistance in cancer cells via various mechanisms. Cancer-derived exosomes could deliver multiple drug resistance-related proteins and miRNAs to specific cells $(30,73)$. Additionally, chemotherapy agents could also be encapsulated and secreted from the extracellular matrix by exosomes, and this mechanism partly accounts for chemotherapy resistance (51).

Much of the focus on cancer therapy concentrates on inhibiting the abnormal proliferation of epithelial cells.
However, this approach has been proven ineffective, with resistance emerging in many types of cancer following adjuvant chemotherapy. Stromal interaction with cancer cells could affect the response to chemotherapy. A detailed hypothesis on the mechanism of resistance has focused on heterotypic interactions, where exosomes are delivered from cancer-associated fibroblasts (CAFs) to pancreatic cancer cells (74). CAFs are inherently insensitive to gemcitabine. When exposed to gemcitabine, pancreatic fibroblasts dramatically increase the release of Snail and miR-146a via exosomes. Utilizing paracrine or potentially an unknown signaling mechanism, these exosomes can be taken up by recipient epithelial cells. During the process, the amount of miR-146a and Snail mRNA in epithelial cells is enhanced. As a result, CAF-derived exosomes facilitate proliferation and chemotherapy resistance. Notably, CAF exosome secretion inhibition could remarkably decrease cell survival, proliferation and drug resistance.

Inhibitors of apoptosis (IAPs) could facilitate cell death, but their expression pattern is decreased in various cancers. In pancreatic cancer, it was confirmed that IAPs are constitutively enhanced by NF- $\mathrm{B}$ in tissues and cell lines (75). This ectopic overexpression of IAPs is associated with cancer progression and chemotherapy resistance. It has been reported that exosomes contain protein and mRNA IAPs (76). When exposed to chemotherapy, the amount of IAP protein or mRNA in the cytoplasm either remained unchanged or was moderately upregulated. Similarly, the expression pattern of IAPs in exosomes exhibited no significant alteration. This stability may partly account for why pancreatic cancer is resistant to chemotherapy (75).

\section{Exosomal miRNAs and proteins for pancreatic cancer diagnosis}

Strong efforts have focused on developing sensitive diagnostics tools improving early detection of pancreatic cancer via identifying pancreatic cancer-associated exosomal markers. The development of next generation sequencing, novel mass spectrometry techniques and new biochemical analyses have rendered the characterization of exosomes more convenient, which means that all exosomal contents have the potential to function as diagnostic biomarkers. Based on a comprehensive literature search, exosomal membrane proteins and miRNAs have provided promising insights into pancreatic cancer progression and tumor burden. Potential exosomal biomarkers for the early detection of pancreatic cancer are summarized in Table I.

A recent study evaluated the expression patterns of four pancreatic cancer-associated miRNAs in circulating exosomes (77). Compared with multiple controls, the expression profile of exosomal miR-21 and miR-17-5p was remarkably enhanced in pancreatic cancer patients, and this distinction could be utilized to discriminate between pancreatic cancer and non-malignant chronic pancreatitis $(\mathrm{CP})$ patients. With the application of this innovative technique, label-free, nanoplasmonic-based short non-coding RNA sensing, a distinctly enhanced amount of exosomal miR-10b was observed in patients with pancreatic cancer compared with $\mathrm{CP}$ or normal controls $(78,79)$. Madhavan et al (80) published a 
Table I. Exosomal biomarkers and early detection of pancreatic cancer.

\begin{tabular}{|c|c|c|c|c|c|}
\hline Disease & Study & Exosome isolation & Exosomal biomarkers & Sensitivity & Specificity \\
\hline \multirow{5}{*}{$\begin{array}{l}\text { Primary pancreatic } \\
\text { cancer }\end{array}$} & Que et al (2013) & Ultracentrifugation & miR-21 & $95.5 \%$ & $81.5 \%$ \\
\hline & (77) & & miR-17-5P & $72.7 \%$ & $92.6 \%$ \\
\hline & $\begin{array}{l}\text { Madhavan et al } \\
(2015)(80)\end{array}$ & $\begin{array}{l}\text { Ultracentrifugation } \\
\text { and immunoaffinity }\end{array}$ & $\begin{array}{l}\text { miR-1246 } \\
\text { miR-4644 } \\
\text { miR-3976 } \\
\text { miR-4306 }\end{array}$ & $100 \%$ & $80 \%$ \\
\hline & $\begin{array}{l}\text { Joshi et al (2015) } \\
\text { (79) }\end{array}$ & LSPR-based assay & miR-10b & $\begin{array}{l}\text { Statistically } \\
\text { significant }\end{array}$ & $\begin{array}{l}\text { Statistically } \\
\text { significant }\end{array}$ \\
\hline & Melo et al (2015) (81) & Ultracentrifugation & GPC1 protein & $100 \%$ & $100 \%$ \\
\hline \multirow[t]{2}{*}{$\begin{array}{l}\text { Pancreatic cancer } \\
\text { liver pre-metastasis }\end{array}$} & $\begin{array}{l}\text { Costa-Silva et al } \\
(2015)(8)\end{array}$ & Ultracentrifugation & MIF & $\begin{array}{l}\text { Statistically } \\
\text { significant }\end{array}$ & $\begin{array}{l}\text { Statistically } \\
\text { significant }\end{array}$ \\
\hline & $\begin{array}{l}\text { Hoshino et al } \\
(2015)(11)\end{array}$ & Ultracentrifugation & Integrins $\alpha v \beta 5$ & $\begin{array}{l}\text { Statistically } \\
\text { significant }\end{array}$ & $\begin{array}{l}\text { Statistically } \\
\text { significant }\end{array}$ \\
\hline
\end{tabular}

LSPR, localized surface plasmon resonance; GPC1, glypican-1; MIF, migration inhibitory factor.

study based on the analysis of five pancreatic cancer-initiating cell markers (CD104, MET, EpCAM, Tspan8 and CD44v6) and four miRNAs (miR-4306, miR-3976, miR-4644 and miR-1246) in serum exosomes, and demonstrated that evaluation of pancreatic cancer-initiating cell markers and miRNA serum-exosome marker panels dramatically enhanced sensitivity (1.00, CI: 0.95-1) with a specificity of 0.80 (CI: 0.67-0.90) for pancreatic cancer vs. all others groups and of 0.93 (CI: 0.81-0.98) excluding non-pancreatic cancer. Concomitant evaluation of these factors distinguished patients with pancreatic cancer from normal controls, CP subjects and patients with benign pancreatic lesions, with $93 \%$ specificity, eliminating non-pancreatic neoplasms (80).

Encouraging advances have been achieved in the search for specific and sensitive biomarkers that could facilitate the early diagnosis of pancreatic cancer. Melo et al (81) reported that glypican-1 (GPC1) expression patterns in exosomes secreted by pancreatic cancer could be utilized to identify subjects with pancreatic cancer early and offer considerable insights into the disease progress and tumor load. A comparison of exosomes from pancreatic cancer and control cell lines indicated that the exosomes from cancer exhibited enhanced levels of GPC1, which has been demonstrated to be upregulated in breast cancer and pancreatic cancer. In serum specimens from subjects with pancreatic cancer $(n=190)$, a significantly larger amount of $\mathrm{GPC}^{+}$circulating exosomes was present compared with normal controls $(n=100)$. Furthermore, $\mathrm{GPCl}^{+}$exosomes were also confirmed to contain identical KRAS mutations, which frequently are present in pancreatic cancer and precancerous lesions and have been considered a fundamental mutation.

Notably, exosomes from patients with intraductal papillary mucinous neoplasm (IPMN; a precancerous lesion of pancreatic cancer) expressed dramatically higher levels of GPC1 than subjects with pancreatitis or cystic adenomas (81). The identification of $\mathrm{GPC}^{+}$circulating exosomes had $100 \%$ specificity in differentiating IPMN from healthy individuals or benign pancreatic disease. When the efficiency of $\mathrm{GPCl}^{+}$circulating exosomes was compared to carbohydrate antigen 19-9 (CA19-9) in differentiating benign from precancerous lesion and healthy subjects, $\mathrm{GPC}^{+}$circulating exosomes was validated to be prominently superior. Tumor burden was associated positively with levels of $\mathrm{GPCl}^{+}$circulating exosomes. In most subjects, the exosome levels reduced following the removal of the solid tumor. A decrease in levels of $\mathrm{GPCl}^{+}$circulating exosomes was associated with the benefit of overall and disease-specific survival in these patients. Additionally, using a genetically engineered mouse that progressively developed into pancreatic cancer, the identification of GPC1+ exosomes exhibited positive results prior to pancreatic lesions being detectable. Another recent paper (11) has reported that integrin $\alpha v \beta 5$-expressing pancreatic cancer exosomes can determine liver metastasis. Targeting integrin $\alpha \mathrm{v} \beta 5$ reduced exosome absorption by resident cells, and inhibited liver metastasis. The levels of integrin $\alpha v \beta 5$ were dramatically enhanced in exosomes isolated from pancreatic cancer subjects with live metastasis compared with subjects with no distant metastasis. Further examination of the molecular mechanisms revealed that integrin $\alpha v \beta 5$ uptake by targeted cells could lead to activating Src phosphorylation and upregulation of pro-inflammatory S100 gene. Collectively, these findings have indicated that $\mathrm{GPC}^{+}$and integrin $\alpha v \beta 5$-expressing circulating exosomes have great potential as indicators of pancreatic cancer progression and organ-specific metastasis in patients.

A schematic of the roles that exosomes may have in pancreatic cancer metastasis and their potential for early detection is summarized in Fig. 2.

\section{Conclusion}

Circulating exosomes have demonstrated potential as reliable candidates for the early diagnosis of pancreatic cancer, for use in screening high-risk individuals without clinical presentation of cancer, and for monitoring the course of the disease. Accumulating evidence has revealed that exosomes can indicate the clinical management of pancreatic cancer, improve overall survival and prevent organ-specific metastasis. Nevertheless, 


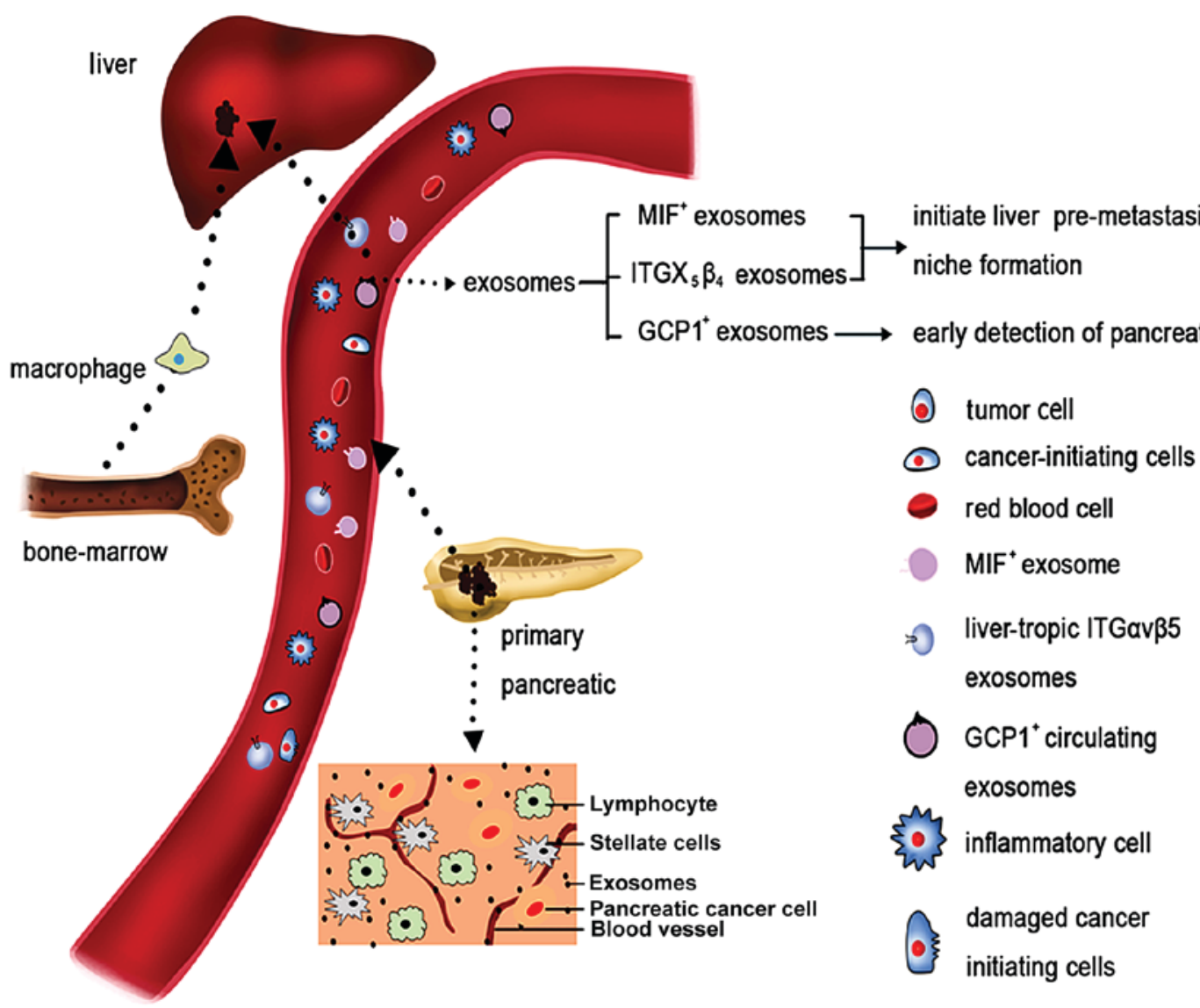

Figure 2. Pancreatic cancer, its microenvironment and its metastatic route. Pancreatic cancer is characterized by a dense stromal reaction, consisting of a desmoplastic reaction with the extracellular matrix, CAFs, tumor cells and immune cells. Both pancreatic cancer cells and CAFs can release exosomes. Exosomes released by pancreatic cancer cells enter the vessels. Due to specific integrin complex expression, exosomes migrate preferentially to the liver. There, they are taken up by Kupffer cells (also known as stellate macrophages), which react by increasing TGF- $\beta$ signaling. Subsequently, macrophage MIF is released from the exosomes, which results in the initiation of an immune-evasive response. This model supports the creation of a metastatic niche for pancreatic cancer cells mediated by exosomes, which is then followed by the establishment of liver metastases. CAFs, cancer-associated fibroblasts; TGF, transforming growth factor; MIF, migration inhibitory factor; ITG, integrin; GCP1, glypican-1.

the clinical application of exosomes in the differential diagnosis of pancreatic cancer requires further research. More studies are needed to better elucidate the dynamic alteration of serous exosomes in the progression of pancreatic cancer, to evaluate the possibility of plasmatic exosomal biomarker panels for high-risk individuals in a large-scale cohort, and understand the cellular and molecular functions of exosomes in pancreatic cancer.

\section{Future perspective}

The prognosis of patients with pancreatic cancer has improved slowly during the past decade, and the low survival rate is due to multiple factors, but, perhaps the predominant determinant of survival is the advanced stage at which most subjects are diagnosed. The development of biomarkers to improve detection at early stages could apply to high-risk individuals, such as those with weight loss and new-onset diabetes. New-onset diabetes and concurrent weight loss have been demonstrated to occur a few months prior to the clinical manifestation of pancreatic cancer (68). Multiple studies have demonstrated that new-onset diabetes of $<3$ years enhances the risk of pancreatic cancer diagnosis 5-7-fold, which is significantly higher than the effect of long-term diabetes, which only accounts for a 1.5 -fold increase in risk within a course of $>5$ years $(82,83)$. The discovery of novel exosome-associated biomarkers for paraneoplastic effects, such as weight loss and new-onset diabetes, will aid in the early diagnosis of pancreatic cancer and improve the quality of life of the patients with the disease.

However, the exact sensitivity and specificity of these discovered biomarkers for disease prescreening in diagnosed individuals remains uncertain. Forinstance, the amountof $\mathrm{GCP} 1^{+}$ circulating exosomes detectable in blood that may be needed to provide enough information for the diagnosis and prognosis of pancreatic cancer remains unknown. The paper published by Costa-Silva et al (8) elaborated on organotropic metastasis mechanisms and emphasized the vital role of exosomes in facilitating cancer metastasis. Furthermore, another recent study demonstrated that pancreatic cancer-derived exosomes could result in increasing liver metastatic load by delivering $\mathrm{MIF}^{+}$exosomes to Kupffer cells and attracting inflammatory cells to elicit the liver pre-metastatic niche formation (84). More studies that further evaluate the association between levels of $\mathrm{MIF}^{+}$exosomes and the progression and diagnosis of the disease are needed. Finally, compared with subjects whose pancreatic cancer has not progressed, exosomal integrin $\alpha v \beta 5$ 
was markedly upregulated in exosomes from patients who eventually developed distant liver metastasis. These findings indicated that exosomal integrin $\alpha \mathrm{v} \beta 5$ and MIF orchestrated organ-specific metastasis and might be reliable biomarkers for the diagnosis of pancreatic cancer and metastatic stage. Particularly, strategies targeting these molecular components could be utilized to prevent organotropic metastasis.

In conclusion, a better understanding of the molecular mechanisms that initiate pre-metastatic niche formation and an evaluation of their features and functions in cancer metastasis will provide novel insights for the early diagnosis and may lead to therapeutic regimens for the treatment of pancreatic cancer.

\section{Acknowledgements}

Not applicable.

\section{Funding}

No funding was received.

\section{Availability of data and materials}

Not applicable.

\section{Author's contributions}

YY performed the literature search and was a major contributor towards writing the manuscript. GF contributed towards writing and critically revising the manuscript and for important intellectual content. LM made contributions to the conception and design of the present review. All authors read and approved the final manuscript.

\section{Ethics approval and consent to participate}

Not applicable.

\section{Consent for publication}

Not applicable.

\section{Competing interests}

The authors declare that they have no competing interests.

\section{References}

1. Siegel RL, Miller KD and Jemal A: Cancer statistics, 2016. CA Cancer J Clin 66: 7-30, 2016.

2. Iacobuzio-Donahue CA: Genetic evolution of pancreatic cancer: Lessons learnt from the pancreatic cancer genome sequencing project. GUT 61: 1085-1094, 2012.

3. Yachida S, Jones S, Bozic I, Antal T, Leary R, Fu B, Kamiyama M, Hruban RH, Eshleman JR, Nowak MA, et al: Distant metastasis occurs late during the genetic evolution of pancreatic cancer. Nature 467: 1114-1117, 2010.

4. György B, Szabó TG, Pásztói M, Pál Z, Misják P, Aradi B, László V, Pállinger E, Pap E, Kittel A, et al: Membrane vesicles, current state-of-the-art: Emerging role of extracellular vesicles Cell Mol Life Sci 68: 2667-2688, 2011.

5. Simons M and Raposo G: Exosomes-vesicular carriers for intercellular communication. Curr Opin Cell Biol 21: 575-581, 2009.
6. Cocucci E and Meldolesi J: Ectosomes and exosomes: Shedding the confusion between extracellular vesicles. Trends Cell Biol 25: 364-372, 2015

7. Cocucci E and Meldolesi J: Ectosomes. Curr Biol 21: R940-R941, 2011.

8. Costa-Silva B, Aiello NM, Ocean AJ, Singh S, Zhang H, Thakur BK, Becker A, Hoshino A, Mark MT, Molina H, et al: Pancreatic cancer exosomes initiate pre-metastatic niche formation in the liver. Nat Cell Biol 17: 816-826, 2015.

9. Christianson HC, Svensson KJ, van Kuppevelt TH, Li JP and Belting M: Cancer cell exosomes depend on cell-surface heparan sulfate proteoglycans for their internalization and functional activity. Proc Natl Acad Sci USA 110: 17380-17385, 2013.

10. Miyanishi M, Tada K, Koike M, Uchiyama Y, Kitamura T and Nagata S: Identification of Tim4 as a phosphatidylserine receptor. Nature 450: 435-439, 2007.

11. Hoshino A, Costa-Silva B, Shen TL, Rodrigues G, Hashimoto A, Tesic Mark M, Molina H, Kohsaka S, Di Giannatale A, Ceder S, et al: Tumour exosome integrins determine organotropic metastasis. Nature 527: 329-335, 2015.

12. Nazarenko I, Rana S, Baumann A, McAlear J, Hellwig A, Trendelenburg M, Lochnit G, Preissner KT and Zöller M: Cell surface tetraspanin Tspan 8 contributes to molecular pathways of exosome-induced endothelial cell activation. Cancer Res 70: 1668-1678, 2010.

13. Zöller M: Exosomes in cancer disease. Methods Mol Biol 1381: 111-149, 2016.

14. Li Y, Zheng Q, Bao C, Li S, Guo W, Zhao J, Chen D, Gu J, He X and Huang S: Circular RNA is enriched and stable in exosomes: A promising biomarker for cancer diagnosis. Cell Res 25: 981-984, 2015.

15. Ge Q, Zhou Y, Lu J, Bai Y, Xie X and Lu Z: miRNA in plasma exosome is stable under different storage conditions. Molecules 19: 1568-1575, 2014.

16. Subra C,Grand D, Laulagnier K, Stella A,Lambeau G,Paillasse M, De Medina P, Monsarrat B, Perret B, Silvente-Poirot S, et al: Exosomes account for vesicle-mediated transcellular transport of activatable phospholipases and prostaglandins. J Lipid Res 51: 2105-2120, 2010

17. Beloribi S, Ristorcelli E, Breuzard G, Silvy F, Bertrand-Michel J, Beraud E, Verine A and Lombardo D: Exosomal lipids impact notch signaling and induce death of human pancreatic tumoral SOJ-6 cells. PLoS One 7: e47480, 2012.

18. Beloribi-Djefaflia S, Siret C and Lombardo D: Exosomal lipids induce human pancreatic tumoral $\mathrm{MiaPaCa}-2$ cells resistance through the CXCR4-SDF-1 $\alpha$ signaling axis. Oncoscience 2: 15-30, 2014.

19. Llorente A, Skotland T, Sylvänne T, Kauhanen D, Róg T, Orłowski A, Vattulainen I, Ekroos K and Sandvig K: Molecular lipidomics of exosomes released by PC-3 prostate cancer cells. Biochim Biophys Acta 1831: 1302-1309, 2013.

20. Minciacchi VR, You S, Spinelli C, Morley S, Zandian M, Aspuria PJ, Cavallini L, Ciardiello C, Reis Sobreiro M, Morello M, et al: Large oncosomes contain distinct protein cargo and represent a separate functional class of tumor-derived extracellular vesicles. Oncotarget 6: 11327-11341, 2015.

21. Keerthikumar S, Gangoda L, Liem M, Fonseka P, Atukorala I, Ozcitti C, Mechler A, Adda CG, Ang CS and Mathivanan S: Proteogenomic analysis reveals exosomes are more oncogenic than ectosomes. Oncotarget 6: 15375-15396, 2015.

22. Lötvall J, Hill AF, Hochberg F, Buzás EI, Di Vizio D, Gardiner C, Gho YS, Kurochkin IV, Mathivanan S, Quesenberry P, et al: Minimal experimental requirements for definition of extracellular vesicles and their functions: A position statement from the International Society for Extracellular Vesicles. J Extracell Vesicles 3: 26913, 2014.

23. Zöller M: Tetraspanins: Push and pull in suppressing and promoting metastasis. Nat Rev Cancer 9: 40-55, 2009.

24. Pols MS and Klumperman J: Trafficking and function of the tetraspanin CD63. Exp Cell Res 315: 1584-1592, 2009.

25. Yue S, Mu W, Erb U and Zöller M: The tetraspanins CD151 and Tspan8 are essential exosome components for the crosstalk between cancer initiating cells and their surrounding. Oncotarget 6: 2366-2384, 2015.

26. Henderson MC and Azorsa DO: The genomic and proteomic content of cancer cell-derived exosomes. Front Oncol 2: 38, 2012.

27. Raimondo F, Morosi L, Chinello C, Magni F and Pitto M: Advances in membranous vesicle and exosome proteomics improving biological understanding and biomarker discovery. Proteomics 11: 709-720, 2011. 
28. Runz S, Keller S, Rupp C, Stoeck A, Issa Y, Koensgen D, Mustea A, Sehouli J, Kristiansen G and Altevogt P: Malignant ascites-derived exosomes of ovarian carcinoma patients contain CD24 and EpCAM. Gynecol Oncol 107: 563-571, 2007.

29. Al-Nedawi K, Meehan B, Micallef J, Lhotak V, May L, Guha A and Rak J: Intercellular transfer of the oncogenic receptor EGFRvIII by microvesicles derived from tumour cells. Nat Cell Biol 10: 619-624, 2008.

30. Corcoran C, Rani S, O'Brien K, O'Neill A, Prencipe M, Sheikh R, Webb G, McDermott R, Watson W, Crown J and O'Driscoll L: Docetaxel-resistance in prostate cancer: Evaluating associated phenotypic changes and potential for resistance transfer via exosomes. PLoS One 7: e50999, 2012.

31. Demory Beckler M, Higginbotham JN, Franklin JL, Ham AJ, Halvey PJ, Imasuen IE, Whitwell C, Li M, Liebler DC and Coffey RJ: Proteomic analysis of exosomes from mutant KRAS colon cancer cells identifies intercellular transfer of mutant KRAS. Mol Cell Proteomics 12: 343-355, 2013.

32. Ji H, Greening DW, Barnes TW, Lim JW, Tauro BJ, Rai A, Xu R, Adda C, Mathivanan S, Zhao W, et al: Proteome profiling of exosomes derived from human primary and metastatic colorectal cancer cells reveal differential expression of key metastatic factors and signal transduction components. Proteomics 13: $1672-1686,2013$

33. Park JA, Sharif AS, Tschumperlin DJ, Lau L, Limbrey R, Howarth P and Drazen JM: Tissue factor-bearing exosome secretion from human mechanically stimulated bronchial epithelial cells in vitro and in vivo. J Allergy Clin Immunol 130: 1375-1383, 2012.

34. Adamczyk KA, Klein-Scory S, Tehrani MM, Warnken U, Schmiegel W, Schnölzer $M$ and Schwarte-Waldhoff I: Characterization of soluble and exosomal forms of the EGFR released from pancreatic cancer cells. Life Sci 89: 304-312, 2011.

35. Salido-Guadarrama I, Romero-Cordoba S, Peralta-Zaragoza O, Hidalgo-Miranda A and Rodríguez-Dorantes M: MicroRNAs transported by exosomes in body fluids as mediators of intercellular communication in cancer. Onco Targets Ther 7: 1327-1338, 2014.

36. Balaj L, Lessard R, Dai L, Cho YJ, Pomeroy SL, Breakefield XO and Skog J: Tumour microvesicles contain retrotransposon elements and amplified oncogene sequences. Nat Commun 2 $180,2011$.

37. Gibbings DJ, Ciaudo C, Erhardt M and Voinnet O: Multivesicular bodies associate with components of miRNA effector complexes and modulate miRNA activity. Nat Cell Biol 11: 1143-1149, 2009.

38. Medina PP, Nolde M and Slack FJ: OncomiR addiction in an in vivo model of microRNA-21-induced pre-B-cell lymphoma. Nature 467: 86-90, 2010

39. Garzon R, Calin GA and Croce CM: MicroRNAs in cancer Annu Rev Med 60: 167-179, 2009.

40. Costinean S, Zanesi N, Pekarsky Y, Tili E, Volinia S, Heerema N and Croce CM: Pre-B cell proliferation and lymphoblastic leukemia/high-grade lymphoma in E(mu)-miR155 transgenic mice. Proc Natl Acad Sci USA 103: 7024-7029, 2006.

41. Mikamori M, Yamada D, Eguchi H, Hasegawa S, Kishimoto T, Tomimaru Y, Asaoka T, Noda T, Wada H, Kawamoto K, et al: MicroRNA-155 controls exosome synthesis and promotes gemcitabine resistance in pancreatic ductal adenocarcinoma. Sci Rep 7: 42339, 2017.

42. Park SM, Gaur AB, Lengyel E and Peter ME: The miR-200 family determines the epithelial phenotype of cancer cells by targeting the E-cadherin repressors ZEB1 and ZEB2. Genes Dev 22: 894-907, 2008.

43. Manier S, Liu CJ, Avet-Loiseau H, Park J, Shi J, Campigotto F, Salem KZ, Huynh D, Glavey SV, Rivotto B, et al: Prognostic role of circulating exosomal miRNAs in multiple myeloma. Blood 129: 2429-2436, 2017.

44. Cortez MA, Bueso-Ramos C, Ferdin J, Lopez-Berestein G, Sood AK and Calin GA: MicroRNAs in body fluids-the mix of hormones and biomarkers. Nat Rev Clin Oncol 8: 467-477, 2011.

45. Boeri M, Verri C, Conte D, Roz L, Modena P, Facchinetti F, Calabrò E, Croce CM, Pastorino U and Sozzi G: MicroRNA signatures in tissues and plasma predict development and prognosis of computed tomography detected lung cancer. Proc Natl Acad Sci USA 108: 3713-3718, 2011.

46. Hansen TB, Jensen TI, Clausen BH, Bramsen JB, Finsen B, Damgaard CK and Kjems J: Natural RNA circles function as efficient microRNA sponges. Nature 495: 384-388, 2013.
47. Memczak S, Jens M, Elefsinioti A, Torti F, Krueger J, Rybak A Maier L, Mackowiak SD, Gregersen LH, Munschauer M, et al: Circular RNAs are a large class of animal RNAs with regulatory potency. Nature 495: 333-338, 2013.

48. Li Q, Shao Y, Zhang X, Zheng T, Miao M, Qin L, Wang B, Ye G, Xiao B and Guo J: Plasma long noncoding RNA protected by exosomes as a potential stable biomarker for gastric cancer. Tumour Biol 36: 2007-2012, 2015.

49. Takahashi K, Yan IK, Kogure T, Haga H and Patel T: Extracellular vesicle-mediated transfer of long non-coding RNA ROR modulates chemosensitivity in human hepatocellular cancer. FEBS Open Bio 4: 458-467, 2014.

50. Mohme M, Riethdorf S and Pantel K: Circulating and disseminated tumour cells-mechanisms of immune surveillance and escape. Nat Rev Clin Oncol 14: 155-167, 2017.

51. Yu S, Cao H, Shen B and Feng J: Tumor-derived exosomes in cancer progression and treatment failure. Oncotarget 6: 37151-37168, 2015.

52. Nguyen DX, Bos PD and Massagué J: Metastasis: From dissemination to organ-specific colonization. Nat Rev Cancer 9: 274-284, 2009.

53. Melo SA, Sugimoto H, O'Connell JT, Kato N, Villanueva A, Vidal A, Qiu L, Vitkin E, Perelman LT, Melo CA, et al: Cancer exosomes perform cell-independent microRNA biogenesis and promote tumorigenesis. Cancer Cell 26: 707-721, 2014.

54. Abd Elmageed ZY, Yang Y, Thomas R, Ranjan M, Mondal D, Moroz K, Fang Z, Rezk BM, Moparty K, Sikka SC, et al: Neoplastic reprogramming of patient-derived adipose stem cells by prostate cancer cell-associated exosomes. Stem Cells 32: 983-997, 2014

55. Falasca M, Kim M and Casari I: Pancreatic cancer: Current research and future directions. Biochim Biophys Acta 1865: 123-132, 2016.

56. Zhang X, Yuan X, Shi H, Wu L, Qian H and Xu W: Exosomes in cancer: Small particle, big player. J Hematol Oncol 8: 83, 2015.

57. Hong BS, Cho JH, Kim H, Choi EJ, Rho S, Kim J, Kim JH, Choi DS, Kim YK, Hwang D and Gho YS: Colorectal cancer cell-derived microvesicles are enriched in cell cycle-related mRNAs that promote proliferation of endothelial cells. BMC Genomics 10: 556, 2009.

58. Le MT, Hamar P, Guo C, Basar E, Perdigão-Henriques R, Balaj L and Lieberman J: miR-200-containing extracellular vesicles promote breast cancer cell metastasis. J Clin Invest 124: 5109-5128, 2014

59. Qu JL, Qu XJ, Zhao MF, Teng YE, Zhang Y, Hou KZ, Jiang YH, Yang XH and Liu YP: Gastric cancer exosomes promote tumour cell proliferation through PI3K/Akt and MAPK/ERK activation. Dig Liver Dis 41: 875-880, 2009.

60. Takikawa T, Masamune A, Yoshida N, Hamada S, Kogure T and Shimosegawa T: Exosomes derived from pancreatic stellate cells: MicroRNA signature and effects on pancreatic cancer cells. Pancreas 46: 19-27, 2017.

61. An M, Lohse I, Tan Z, Zhu J, Wu J, Kurapati H, Morgan MA, Lawrence TS, Cuneo KC and Lubman DM: Quantitative proteomic analysis of serum exosomes from patients with locally advanced pancreatic cancer undergoing chemoradiotherapy. J Proteome Res 16: 1763-1772, 2017.

62. Liu Y, Gu Y and Cao X: The exosomes in tumor immunity. Oncoimmunology 4: e1027472, 2015.

63. Zhou M, Chen J, Zhou L, Chen W, Ding G and Cao L: Pancreatic cancer derived exosomes regulate the expression of TLR4 in dendritic cells via miR-203. Cell Immunol 292: 65-69, 2014.

64. Ding G, Zhou L, Qian Y, Fu M, Chen J, Chen J, Xiang J, Wu Z, Jiang G and Cao L: Pancreatic cancer-derived exosomes transfer miRNAs to dendritic cells and inhibit RFXAP expression via miR-212-3p. Oncotarget 6: 29877-29888, 2015.

65. Wynn TA, Chawla A and Pollard JW: Macrophage biology in development, homeostasis and disease. Nature 496: 445-455, 2013.

66. Su MJ, Aldawsari $\mathrm{H}$ and Amiji M: Pancreatic cancer cell exosome-mediated macrophage reprogramming and the role of MicroRNAs 155 and $125 \mathrm{~b} 2$ transfection using nanoparticle delivery systems. Sci Rep 6: 30110, 2016.

67. Katsiougiannis S, Chia D, Kim Y, Singh RP and Wong DT: Saliva exosomes from pancreatic tumor-bearing mice modulate NK cell phenotype and antitumor cytotoxicity. FASEB J 31: 998-1010,2017.

68. Kamisawa T, Wood LD, Itoi T and Takaori K: Pancreatic cancer. Lancet 388: 73-85, 2016. 
69. Sagar G, Sah RP, Javeed N, Dutta SK, Smyrk TC, Lau JS Giorgadze N, Tchkonia T, Kirkland JL, Chari ST and Mukhopadhyay D: Pathogenesis of pancreatic cancer exosome-induced lipolysis in adipose tissue. Gut 65: 1165-1174, 2016.

70. Javeed N, Sagar G, Dutta SK, Smyrk TC, Lau JS, Bhattacharya S, Truty M, Petersen GM, Kaufman RJ, Chari ST and Mukhopadhyay D: Pancreatic cancer-derived exosomes cause paraneoplastic $\beta$-cell dysfunction. Clin Cancer Res 21: $1722-1733,2015$.

71. Patel GK, Patton MC, Singh S, Khushman M and Singh AP: Pancreatic cancer exosomes: Shedding off for a meaningful journey. Pancreat Disord Ther 6: e148, 2016.

72. Oettle H, Post S, Neuhaus P, Gellert K, Langrehr J, Ridwelski K, Schramm H, Fahlke J, Zuelke C, Burkart C, et al: Adjuvant chemotherapy with gemcitabine vs observation in patients undergoing curative-intent resection of pancreatic cancer: A randomized controlled trial. JAMA 297: 267-277, 2007.

73. Wei Y, Lai X, Yu S, Chen S, Ma Y, Zhang Y, Li H, Zhu X, Yao L and Zhang J: Exosomal miR-221/222 enhances tamoxifen resistance in recipient ER-positive breast cancer cells. Breast Cancer Res Treat 147: 423-431, 2014

74. Richards KE, Zeleniak AE, Fishel ML, Wu J, Littlepage LE and Hill R: Cancer-associated fibroblast exosomes regulate survival and proliferation of pancreatic cancer cells. Oncogene 36 : 1770-1778, 2017

75. Asuncion Valenzuela MM, Castro I, Gonda A, Diaz Osterman CJ Jutzy JM, Aspe JR, Khan S, Neidigh JW and Wall NR: Cell death in response to antimetabolites directed at ribonucleotide reductase and thymidylate synthase. Onco Targets Ther 8: 495-507, 2015.

76. Valenzuela MM, Ferguson Bennit HR, Gonda A, Diaz Osterman CJ, Hibma A, Khan S and Wall NR: Exosomes secreted from human cancer cell lines contain Inhibitors of Apoptosis (IAP). Cancer Microenviron 8: 65-73, 2015.

77. Que R, Ding G, Chen J and Cao L: Analysis of serum exosomal microRNAs and clinicopathologic features of patients with pancreatic adenocarcinoma. World J Surg Oncol 11: 219 , 2013.
78. Lu L and Risch HA: Exosomes: Potential for early detection in pancreatic cancer. Future Oncol 12: 1081-1090, 2016.

79. Joshi GK, Deitz-McElyea S, Liyanage T, Lawrence K, Mali S, Sardar R and Korc M: Label-Free nanoplasmonic-based short noncoding RNA sensing at attomolar concentrations allows for quantitative and highly specific assay of MicroRNA-10b in biological fluids and circulating exosomes. ACS Nano 9: 11075-11089, 2015

80. Madhavan B, Yue S, Galli U, Rana S, Gross W, Müller M, Giese NA, Kalthoff H, Becker T, Büchler MW and Zöller M: Combined evaluation of a panel of protein and miRNA serum-exosome biomarkers for pancreatic cancer diagnosis increases sensitivity and specificity. Int J Cancer 136: 2616-2627, 2015.

81. Melo SA, Luecke LB, Kahlert C, Fernandez AF, Gammon ST, Kaye J, LeBleu VS, Mittendorf EA, Weitz J, Rahbari N, et al: Glypican-1 identifies cancer exosomes and detects early pancreatic cancer. Nature 523: 177-182, 2015.

82. Ben Q, Xu M, Ning X, Liu J, Hong S, Huang W, Zhang H and Li Z: Diabetes mellitus and risk of pancreatic cancer: A meta-analysis of cohort studies. Eur J Cancer 47: 1928-1937, 2011.

83. Huxley R, Ansary-Moghaddam A, Berrington de González A Barzi $\mathrm{F}$ and Woodward M: Type-II diabetes and pancreatic cancer: A meta-analysis of 36 studies. Br J Cancer 92: 2076-2083, 2005.

84. Liu Y and Cao X: Organotropic metastasis: Role of tumor exosomes. Cell Res 26: 149-150, 2016.

(i) $(-)$ This work is licensed under a Creative Commons Attribution-NonCommercial-NoDerivatives 4.0 International (CC BY-NC-ND 4.0) License. 